\title{
A CASE STUDY ON COMPARATIVE ANALYSIS OF 3D POINT CLOUDS FROM UAV MOUNTED AND TERRESTRIAL SCANNERS FOR BRIDGE CONDITION ASSESSMENT
}

\author{
Varun Kasireddy ${ }^{1}$, and Burcu Akinci ${ }^{2}$
}

\begin{abstract}
Recent improvements in 3D laser scanning technologies enabled accurate capturing of existing spatial conditions using point clouds. To leverage this progress for a more accurate condition assessment, state Department of Transportations (DOT) across the US have been utilizing scanning technologies through deployment of terrestrial laser scanners (TLS) and more recently Light Detection and Ranging (LIDAR) scanners fitted to UAVs. However, not much study thus far discusses the potential of these 3D point clouds captured from these two different data collection approaches for supporting automatic and detailed (element-level) condition assessment of bridges. The goal of this study is to compare the utilization of 3D point cloud generated from a terrestrial scanner with that from a UAV in supporting element-level condition assessment of bridges. This paper presents recent results from full scale deployment on a small/medium size highway bridge. An important component of our effort is to explore how well the collected data supports element level condition assessment as per National Bridge Inspection Standards (NBIS). This study assesses the potential to incorporate these data collection modalities into standard bridge safety inspection practice.
\end{abstract}

Keywords: Laser scanning, bridge, condition assessment, element level.

\section{INTRODUCTION}

Recent ASCE Report Card for America’s infrastructure (ASCE 2013) indicated the average grade for bridges to be C+. Given the sheer number of bridges in the US $(>600,000)$ and the substandard grade, it is necessary to emphasize the importance of assessing current conditions and frequent monitoring of the health of bridges. Current routine inspection requires sending inspectors to observe, measure and record defects manually, which is labour-intensive and dangerous. Also, this inspection process usually requires shutting down a portion of or even all of a bridge structure's daily operation, which result in inconvenience to surrounding communities (Koch et al. 2015; Zhu \& Brilakis 2010). Results recorded in this way can also be error-prone because they are subject to transcription errors, measurement errors and they highly depend on personal judgement of the inspector (Phares et al. 2004). To overcome some of these issues, there are ongoing efforts to explore scanning technologies, ground based as well as those fitted to UAV, to capture geometric information about bridges in addition to routine bridge inspection (Yan et al. 2016). While there have been several applications for bridges using point clouds, such

1 Ph.D. Candidate, Department of Civil \& Environmental Engineering, Carnegie Mellon University, Pittsburgh, PA, varunkasi@cmu.edu

2 Paul P. Christiano Professor, Department of Civil \& Environmental Engineering, Carnegie Mellon University, Pittsburgh, PA, bakinci@cmu.edu 
as assessing a bridge's surface flatness (Tang et al. 2009) and under clearance (Tang \& Akinci 2012a; Riveiro et al. 2013), and for supporting visual bridge inspection (Kasireddy \& Akinci 2015), there is currently no known initiative by the DOTs to exploit scan data for in-depth element-level condition assessment.

Prior to attempting to use scan data for element-level condition assessment, it is necessary to understand requirements of such an assessment as specified by NBIS. As per these standards, an inspector assesses condition of quantities of bridge elements into one of the four condition states (CS1 - Good; CS2 - Fair; CS3 - Poor; CS4 - Severe). These standards provide a clear classification of defects based on material type. For example, concrete elements have defects, such as cracks, spalling and efflorescence; whereas, steel elements have defects, such as section loss, rusting, and distortion. The standards also specify what would constitute different condition levels of each of these defects.

In practice, during condition assessment, inspectors first identify all instances of different types of defects present on all elements of a bridge. Then, they use several characteristics of the defect instance, such as spatial extent, severity and colour, to compare against reference values provided for each condition state in the element inspection specifications to assign the defect instance to an appropriate condition state (AASHTO 2013). Further, this procedure is repeated for all the instances to accumulate quantities for different condition states of all bridge elements under consideration.

While point clouds can be leveraged to access information relating to characteristics of a defect instance, manually extracting such information from point clouds can be tedious and error prone (Tang \& Akinci 2012a). Therefore, in the context of using scans for condition assessment, if these specifications are formalized in a computer-interpretable manner, then it can support automated element-level condition assessment. Here, it can be either a complete condition assessment, or a sub task such as, preliminary analysis using point cloud features to check if the collected scans can allow detection and assessment of defects of certain types and of various condition state levels (when they are present). Specifically, the focus of this paper is on the latter one. Such a preliminary analysis would help get a quick sense of the quality of data at hand, before delving into a detailed condition assessment of every defect instance. As TLS and UAV mounted scanners are increasingly being considered for use in bridge inspection and assessment practice, we propose quantitative approaches on scans collected from these scanners to understand their suitability to condition assessment.

\section{CASE DESCRIPTION}

In this case study, we collected scan data at a bridge site using TLS and UAV mounted scanners (UMS), and merged scans collected by these devices separately to get a complete 3D point cloud representation of the bridge. Then, we compared point clouds generated from these two devices to determine how much they support element-level condition assessment.

\subsection{Overview of the bridge}

Bridge A built over a creek in 1937 has a superstructure comprised of two simple steel multi-girder spans. The reinforced concrete deck width measures 33.2 feet (10.12 meters) outer-edge to outer-edge, with tar-and-chip overlay surface. The substructure consists of two reinforced concrete abutments and one reinforced concrete pier. While examining the condition commentary recorded in the inspection reports, we observed that this bridge 
contains instances of paint loss, concrete delamination/ spalling, and steel corrosion (with or without section loss).

\subsection{Scanner configuration and sensors}

To collect scan data for this study, we used a medium range commercially available phasebased scanner as the TLS option, whereas the UAV has been fitted with state-of-the-art sensors that are required to enable both, mapping and localization. Figure 1 shows pictures of both scanners and Figure 2a shows scanner locations and representative flight paths during data collection of this bridge. For data collection, our goal was to position the TLS in a manner to ensure capturing maximum amount of visibly exposed bridge element surfaces. With similar data collection goals, UAV path included at least one pass under each bay of the superstructure. The data collected by TLS are stitched together using feature-based registration process, whereas the UAV scans are timestamped as they are being captured, and on-board processing capabilities on UAV are leveraged to register these scans. Overall, the size of the point cloud from TLS is about 12 million points. On the other hand, the point cloud from the UAV scans had approximately 4.8 million points. The top view of Span 1 in these registered scans is given in Figure $2 \mathrm{~b}$.
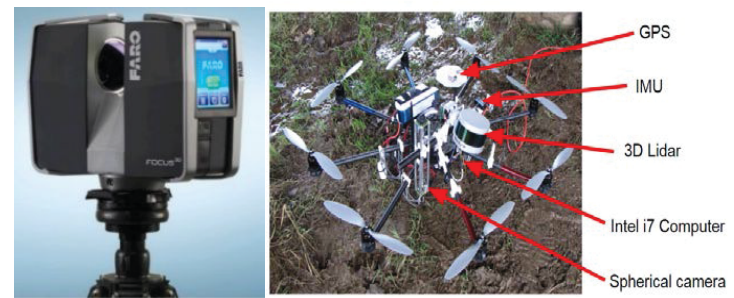

Figure 1: Figure depicting scanners (a) TLS (b) UAV

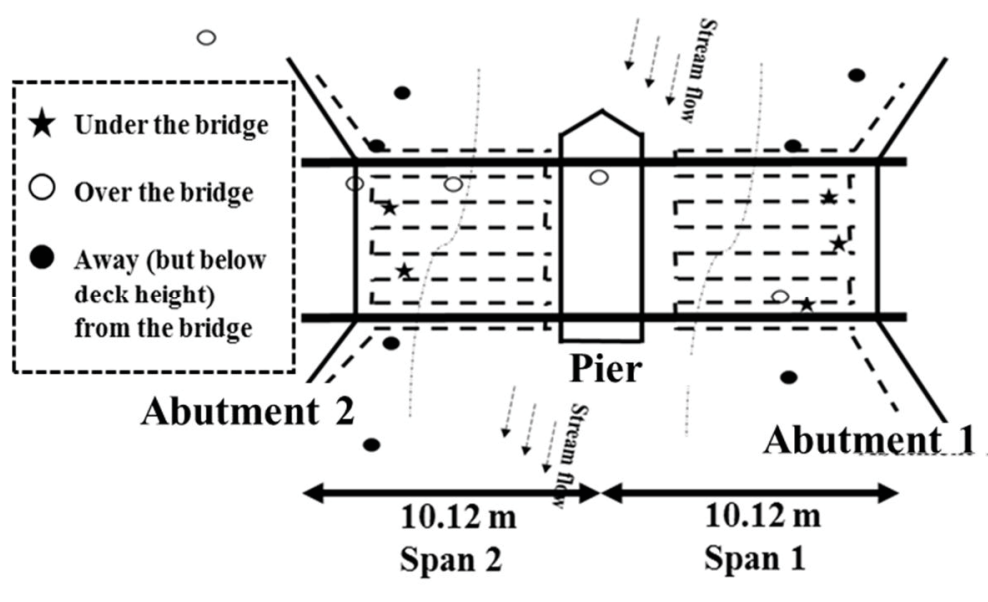

(a)
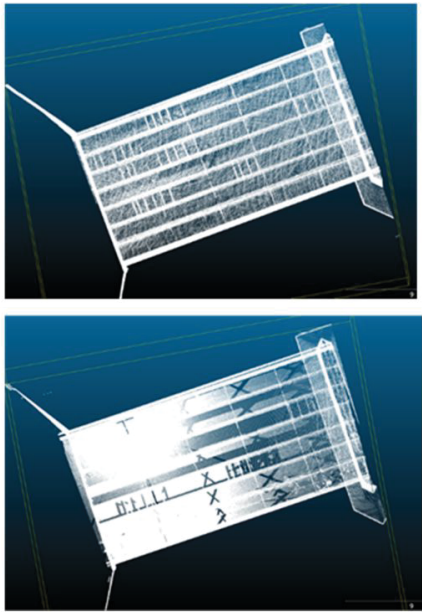

(b)

Figure 2: (a) Figure showing TLS scanner locations and a representative UAV flight path (b) UAV scan (top); Terrestrial Laser scan (below) 
A Case Study on Comparative Analysis of 3D Point Clouds From UAV Mounted And Terrestrial Scanners For Bridge Condition Assessment

\section{APPROACH}

\subsection{Defect scale extraction}

The main goal of this paper is to explore how well scan data supports element level condition assessment as per NBIS. Therefore, to analyze the scan data as per these specifications, we should first go through defect specifications for limit values at which assessment of a condition changes from one state to another.

We use an example to explain these limit values. For instance, specifications for "Spall" defects, shown in Table 1, provide a value for defect properties at which condition states change. In this case, 1-inch threshold for depth of a spall decides if a condition is CS2 or CS3. However, in some cases, specifications can be vague. For example, to designate a condition as CS1, the specification does not provide any value, and it merely indicates "None". This is also in overlap with the specification for CS2 assignment, which states that any spall depth below 1-inch is to be designated CS2. At the same time, it is not practical to assume 0 -inch as the threshold for CS1 as site conditions are rarely perfect. To resolve this overlap, we observed inspectors during a real bridge inspection, and found that inspectors look for spall depth to be at least 1/16th of an inch to classify the condition as CS2, anything below that to be CS1. Therefore, 1/16th of an inch is a limit value for CS1 and CS2. Similarly, for crack width, the limit values are 0.012 inch for CS1 and CS2, and 0.05 for CS2 and CS3. CS4 is a special condition that depends not just on the spatial extent of the defect properties, but also on the defect context, such as the criticality of the element it is present on, and whether it can cause a safety or a structural hazard.

Table 1: Table showing defect properties as per AASHTO Bridge Element Inspection criteria (AASHTO 2013); limit values are highlighted (bold and italics)

\begin{tabular}{|c|c|c|c|c|}
\hline \multirow[t]{3}{*}{ Defects } & \multicolumn{4}{|c|}{ Reinforced Concrete Condition State (CS) Definitions } \\
\hline & CS - 1 & CS - 2 & CS - 3 & $\mathrm{CS}-4$ \\
\hline & GOOD & FAIR & POOR & SEVERE \\
\hline \begin{tabular}{|l|} 
Spalls/ \\
Delamination/ \\
Patch Areas \\
$(1080)$
\end{tabular} & None. & $\begin{array}{l}\text { Delaminated. } \\
\text { Spall } 1 \text { in. or less } \\
\text { deep or } 6 \text { in. or } \\
\text { less in diameter. } \\
\text { Patched area that } \\
\text { is sound. }\end{array}$ & $\begin{array}{l}\text { Spall greater than } 1 \text { in. } \\
\text { deep or greater than } \mathbf{6} \text { in. } \\
\text { diameter. Patched area } \\
\text { that is unsound or showing } \\
\text { distress. Does not warrant } \\
\text { structural review. }\end{array}$ & $\begin{array}{l}\text { The condition warrants a } \\
\text { structural review to determine } \\
\text { the effect on strength or } \\
\text { serviceability of the element or } \\
\text { bridge; OR a structural review } \\
\text { has been completed and the }\end{array}$ \\
\hline $\begin{array}{l}\text { Crack } \\
(1130)\end{array}$ & $\begin{array}{l}\text { Width less than } \\
\text { 0.012 in. or } \\
\text { spacing greater } \\
\text { than } 3.0 \text { ft. }\end{array}$ & $\begin{array}{l}\text { Width } 0.012-0.05 \\
\text { in. or spacing of } \\
1.0-3.0 \mathrm{ft} \text {. }\end{array}$ & $\begin{array}{l}\text { Width greater than } \mathbf{0 . 0 5} \\
\text { in. or spacing of less than } \\
\mathbf{1} \mathbf{f t .}\end{array}$ & $\begin{array}{c}\text { defects impact strength or } \\
\text { serviceability of the element or } \\
\text { bridge }\end{array}$ \\
\hline
\end{tabular}

\subsection{Plane extraction and point cloud assessment}

To do comparative analysis of TLS and UMS scans, we use two features: Point-Plane Distance (PPD) score and Grid Neighbourhood Distance (GND) score. The steps in the process to compute these features from the scan data are shown in Figure 4. Comparing these feature values of both the scans gives us a quick understanding of how well we can do element-level condition assessment using the scans.

\subsubsection{Point-Plane Distance (PPD) score}

For the computation of PPD score, the raw point cloud data is first imported into MATLAB. Then, point cloud is segmented as per bridge element definitions, using techniques, such 
as the one described in (Tang \& Akinci 2012b). These segmented regions were further divided into separate planar sections. Thereafter, Maximum Likelihood Estimation SAmple Consensus (MLESAC) estimator (Torr \& Zisserman 2000), which is a generalization of Random Sample Consensus (RANSAC) estimator, is used to fit a computational plane to the points from these sections. In the next step, "out-of-plane" perpendicular distance between each point and the plane is used to filter points when compared against different defect size scale values. These defect sizes are the limit values of the defect condition states as determined in Section 3.1 (see also Table 1). For each of the scale, PPD score is computed, which is the proportion of points representing the segmented plane that contribute to extraction of the computational plane. We observed that if at least $90 \%$ of the point contribute, then it is possible to quantify defects below the corresponding defect size scale. The results from the comparison of PPD score of TLS and UMS scans are given in the next section. Overall, PPD score provides a measure for checking the ability of scans to support element-level assessment of defects which are primarily out-of-plane with the element surface.

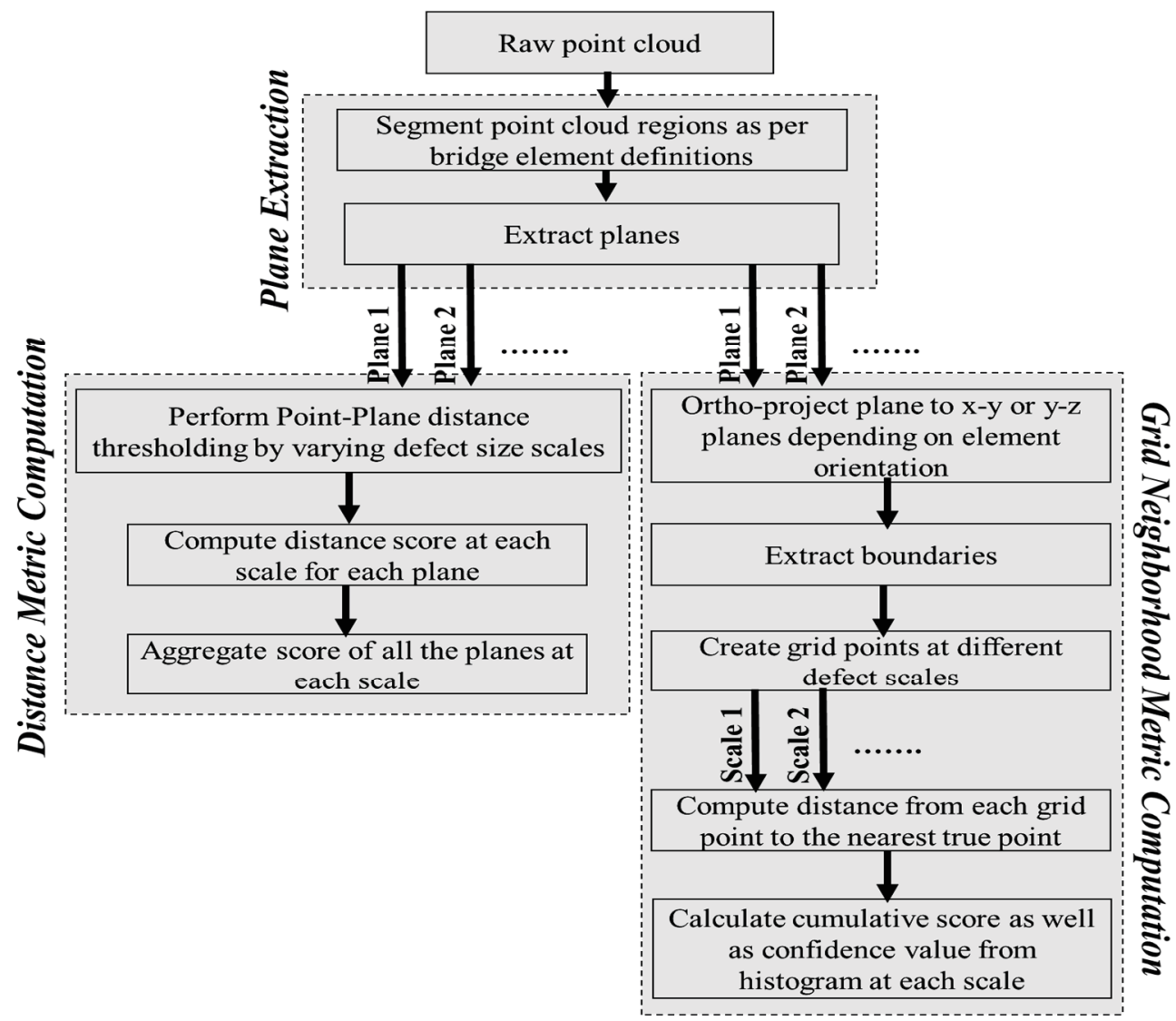

Figure 4: Figure showing the process for performing quantitative assessment of point clouds

\subsubsection{Grid Neighbourhood Distance (GND) score}

In the case of GND score, we follow similar steps as in PPD score computation, until the plane extraction stage. Depending on the orientation of the normal for an element (vertical or horizontal), the points in the segmented plane are ortho-projected, i.e. vertical planes to $y-z$ plane and horizontal planes to $x-y$ plane. Thereafter, the boundaries of the points on these ortho planes are computed. Further, grid points, with spacing equivalent to different defect scales, are created within this boundary. In the next step, distance between each 
grid point to the nearest true point is calculated. GND score represents cumulative distance obtained for all the grid points. This means that higher the score, lower the point density, which can hinder quantifying defects of certain scales. For comparing the score of TLS and UMS scan data, it is necessary to normalize the score with the total number of points in the segmented plane region. An important limitation that should be noted is the effect of outlier points on the boundary computation. Sometimes, this can result in creation of a large number of extra grid points, which can corrupt the estimate of GND score. Overall, the main advantage of this feature is that, it provides a measure of in-plane checking for granularity to support element-level condition assessment. Another way to compute GND score could be by taking histogram of individual distances, which tells us how scattered the true points are, which in turn implicitly indicates the quality of point cloud in its ability to support quantification of different defect sizes. However, this alternate way is out of scope for this paper, and will be discussed in detail in future publications.

\section{RESUlTS AND DisCUSSION}

Due to space constraints, results have been demonstrated only for abutment and deck elements taking the case of a spalling depth. However, the process is the same for other elements and measurable defect properties. As discussed in Section 3.1 (see also Table 1), while assigning condition ratings specific to spalling depth, we observed that $1 / 16$ inch is a limit point between CS1 and CS2, whereas, 1 inch is a limit point between CS2 and CS3. For that reason, we used these values for grid sizes.

From Table 2, it can be observed that for both abutment and deck elements, when a grid size of 1 inch is tested, for TLS, the PPD score is consistently above $90 \%$, which indicates the possibility of detection of CS2, CS3 as well as CS4 condition state defects for spalling. The premise for this is that if most of the points fall under the tolerance limit, any defect greater than the limit can stand a chance for detection. However, if significant number of points fall outside the limit, it is likely the case that the points representing the actual defect will be overshadowed amongst the points representing non-defect surface that are affected by noise. On similar lines of argument, for UMS data, it is possible to detect only CS3 and CS4 level defects, given that PPD score is around $80 \%$ for all the planes. In addition to implying that setting a higher tolerance limit naturally sees increase in the PPD score, setting higher limit also signifies that we are now evaluating for defects at higher severity, i.e. CS3 and CS4. That said, it is also important to note that local effects (e.g. small local regions having no points as opposed to sparse distribution of point across the plane) are not effectively highlighted by this metric. In summary, UMS scans have potential to detect only CS3 and CS4 level out-of-plane defects. On the other hand, we can also detect CS2 level out-of-plane defects with TLS if their dimensions are closer to the limit value separating CS2 and CS3.

Table 3 shows results of GND score computation for Abutment and Deck elements (shown for 1/8" grid size). As discussed in Section 3.2, higher the GND score, lower is the potential to detect and quantify defects from that point cloud. Clearly, for the Abutment section, UMS scan has much higher GND score, as compared to that of TLS scan. This is possibly because, even though spatial distribution of points in the UMS scan was uniform across the element when compared to TLS scan, the point density was much lower than in the case of UMS scan. Conversely, though TLS scans had more occlusions (as seen in Figure $2 \mathrm{~b}$ ) where points representing cross bracings are missing), where points were actually present, and the point density was much higher than that for UMS scans. As a result, we observed lower cumulative distances between the scan points and the grid points. 
Overall, the results indicate that higher the PPD score better are the chances of detecting out-of-plane defects of different scales. Likewise, in the case of GND score, lower score indicates easier detection of in-plane defects, as well as, better distinction among different condition states. These features give an opportunity to quickly test any scan data for feasibility to do element-level condition assessment for different defects and scale levels, and this has been demonstrated using scan data from TLS and UMS.

Table 2 - Results from PPD tests

\begin{tabular}{|c|c|c|c|c|c|c|c|c|}
\hline & & & & & & TLS & & $\mathbf{A V}$ \\
\hline Element & Plane & Unit & Defect & Grid size & PPD score & $\begin{array}{c}\text { Detectable } \\
\text { Condition States }\end{array}$ & PPD score & $\begin{array}{c}\text { Detectable } \\
\text { Condition } \\
\text { States }\end{array}$ \\
\hline Abutment & & LF & Spalling Depth & & & & & \\
\hline & Wing 1 & & & $1 / 16$ inch & $36.32 \%$ & - & $8.50 \%$ & - \\
\hline & & & & 1 inch & $94.45 \%$ & $\mathrm{CS} 2, \mathrm{CS} 3, \mathrm{CS} 4$ & $78.98 \%$ & $\mathrm{CS} 3, \mathrm{CS} 4$ \\
\hline & Wing 2 & & & $1 / 16$ inch & $36.44 \%$ & - & $10.00 \%$ & - \\
\hline & & & & 1 inch & $98.02 \%$ & $\mathrm{CS} 2, \mathrm{CS} 3, \mathrm{CS} 4$ & $83.60 \%$ & CS3, CS4 \\
\hline & Center & & & $1 / 16$ inch & $27.19 \%$ & - & $8.20 \%$ & - \\
\hline & & & & 1 inch & $93.63 \%$ & $\mathrm{CS} 2, \mathrm{CS} 3, \mathrm{CS} 4$ & $80.49 \%$ & $\mathrm{CS} 3, \mathrm{CS} 4$ \\
\hline Deck & & SF & Spalling Depth & & & & & \\
\hline & Top & & & $1 / 16$ inch & $34.85 \%$ & - & $9.01 \%$ & \\
\hline & & & & 1 inch & $92.19 \%$ & $\mathrm{CS} 2, \mathrm{CS} 3, \mathrm{CS} 4$ & $81.59 \%$ & CS3, CS4 \\
\hline
\end{tabular}

Table 3 - Results from GND tests

\begin{tabular}{|l|l|l|l|l|l|l|}
\hline \multicolumn{9}{|l|}{ Element } & Plane & Unit & Defect & Grid size & $\begin{array}{l}\text { Cumulative GND } \\
\text { score }\end{array}$ & $\begin{array}{l}\text { Cumulative GND } \\
\text { score }\end{array}$ \\
\hline Abutment & & LF & Spalling Depth & & & \\
\hline & Wing 1 & & & $1 / 8$ inch & 0.0795 & 0.1012 \\
\hline & Wing 2 & & & $1 / 8$ inch & 0.0390 & 0.0859 \\
\hline & Center & & & $1 / 8$ inch & 0.0103 & 0.1409 \\
\hline Deck & & SF & Spalling Depth & & & \\
\hline & Top & & & $1 / 8$ inch & 0.0303 & 0.0014 \\
\hline
\end{tabular}

\section{CONCLUSIONS}

Current manual approach for inspection and condition assessment are tedious and error prone, and these issues can potentially be addressed with reality capture technology, such as laser scanning. This paper describes a case study to do a comparative analysis to understand the feasibility of TLS and UMS scans for element-level condition assessment.

To quickly compare the scans obtained via TLS and UMS in a quantitative manner, two features were used: PPD score for out-of-plane defects and GND score for in-plane defects. Results from the analysis suggest that UMS scans are currently capable of supporting only CS3 and CS4 level defects. TLS scans additionally help distinguish between CS2 and CS3 level defects.

The limitation of this study is that the potential of scans is judged based solely on 3D spatial data. In other situations, information such as colour and intensity can provide valuable insights about the potential to defect and quantify certain type of defects, such as rusting and efflorescence among others. In the future work, we will focus on these aspects to achieve a more complete evaluation of point clouds. In addition to that, we will do 
process analyses of using these two modes of scanning to better quantify the benefits and impact on condition assessment of bridges. The limitations of the scans (e.g. occlusions and outlier points near scan boundaries) notwithstanding, the results presented demonstrate the potential of scans to be used for automatically detecting and quantifying defects of higher condition state levels, and serve as complement to human inspections.

\section{ACKNOWLEDGMENTS}

The project is funded by a grant from the National Science Foundation (NSF), \#1328930. NSF's support is greatly acknowledged. The authors also acknowledge the kind support from project collaborators, Dr. Scherer and Luke Yoder for sharing the data collected by UAV during field studies, and Michael Baker International and Pennsylvania Department of Transportation, for giving permission to scan the bridge. Any opinions, findings, conclusions or recommendations presented in this paper are those of authors and do not necessarily reflect the views of the NSF and others listed in this section.

\section{REFERENCES}

AASHTO, 2013. Manual for Bridge Element Inspection, AASHTO.

ASCE, 2013. 2013 Report Card for America's Infrastructure, Reston, VA: American Society for Civil Engineers.

Kasireddy, V. \& Akinci, B., 2015. Towards the integration of inspection data with bridge information models to support visual condition assessment. In Congress on Computing in Civil Engineering, Proceedings. Austin, Texas: American Society of Civil Engineers (ASCE), pp. 644-651.

Koch, C. et al., 2015. Advanced Engineering Informatics A review on computer vision based defect detection and condition assessment of concrete and asphalt civil infrastructure. Advanced Engineering Informatics, 29(2), pp.196-210.

Phares, B.M. et al., 2004. Routine Highway Bridge Inspection Condition Documentation Accuracy and Reliability. Journal of Bridge Engineering, 9(4), pp.403-413.

Riveiro, B. et al., 2013. Validation of terrestrial laser scanning and photogrammetry techniques for the measurement of vertical underclearance and beam geometry in structural inspection of bridges. Measurement, 46(1), pp.784-794.

Tang, P. \& Akinci, B., 2012a. Automatic execution of workflows on laser-scanned data for extracting bridge surveying goals. Advanced Engineering Informatics, 26(4), pp.889903.

Tang, P. \& Akinci, B., 2012b. Formalization of workflows for extracting bridge surveying goals from laser-scanned data. Automation in Construction, 22, pp.306-319.

Tang, P., Akinci, B. \& Huber, D., 2009. Characterization of three algorithms for detecting surface flatness defects from dense point clouds. In IS\&T/SPIE Electronic Imaging. p. $72390 \mathrm{~N}-72390 \mathrm{~N}$.

Torr, P.H.S. \& Zisserman, A., 2000. MLESAC: A New Robust Estimator with Application to Estimating Image Geometry. Computer Vision and Image Understanding, 78(1), pp.138-156.

Yan, Y. et al., 2016. Automated Damage Detection and Structural Modelling with Laser Scanning. In Proceedings of the Structural Stability Research Council Annual Stability Conference. Chicago, Illinois: Structural Stability Research Council, pp. 842-861.

Zhu, Z. \& Brilakis, I., 2010. Concrete column recognition in images and videos. Journal of computing in civil engineering, 24(6), pp.478-487. 